\title{
Latin American science and technology in a global context
}

Selected papers presented at the VIII International Seminar on the Quantitative and Qualitative Study of Science and Technology "Prof. Gilberto Sotolongo Aguilar" on 2-3 November 2016.

Guest Editors:

Jane M. Russell (Mexico) and María-Victoria Guzmán (Cuba). 